\title{
Temporal wind pairs for space launch vehicle capability assessment and risk mitigation
}

\author{
Ryan K. Decker ${ }^{1}$ \\ NASA/Marshall Space Flight Center, Huntsville, AL, 35812
}

Robert E. Barbré, Jr. ${ }^{2}$

Jacobs, Huntsville, AL, 35806

\begin{abstract}
Space launch vehicles incorporate upper-level wind assessments to determine wind effects on the vehicle and for a commit to launch decision. These assessments make use of wind profiles measured hours prior to launch and may not represent the actual wind the vehicle will fly through. Uncertainty in the winds over the time period between the assessment and launch introduces uncertainty in assessment of vehicle controllability and structural integrity that must be accounted for to ensure launch safety. Temporal wind pairs are used in engineering development of allowances to mitigate uncertainty. Five sets of temporal wind pairs at various times (0.75, 1.5, 2, 3 and 4-hrs) at the United States Air Force Eastern Range and Western Range, as well as the National Aeronautics and Space Administration's Wallops Flight Facility are developed for use in upper-level wind assessments on vehicle performance. Historical databases are compiled from balloon-based and vertically pointing Doppler radar wind profiler systems. Various automated and manual quality control procedures are used to remove unacceptable profiles. Statistical analyses on the resultant wind pairs from each site are performed to determine if the observed extreme wind changes in the sample pairs are representative of extreme temporal wind change. Wind change samples in the Eastern Range and Western Range databases characterize extreme wind change. However, the small sample sizes in the Wallops Flight Facility databases yield low confidence that the sample population characterizes extreme wind change that could occur.
\end{abstract}

\section{Nomenclature}

$k \quad=$ scale parameter of the Generalized Extreme Value estimate

$P_{u} \quad=$ probability level of a universal population

$P_{s} \quad=$ probability level of a sample population

$n \quad$ = sample size

$u$ and $v \quad=$ east-west and north-south wind change components, respectively, $\mathrm{m} / \mathrm{s}$

$x \quad=$ value of wind change in Generalized Extreme Value probability distribution function, $\mathrm{m} / \mathrm{s}$

$y=$ probability level in Generalized Extreme Value probability distribution function

$\mu \quad=$ shape parameter of the Generalized Extreme Value estimate

$\sigma \quad=$ location parameters of the Generalized Extreme Value estimate

\section{Introduction}

Space launch vehicle commit-to-launch decisions include an assessment of the upper-level (UL) atmospheric wind environment to determine the vehicle's predicted controllability and structural integrity

\footnotetext{
${ }^{1}$ Aerospace Technologist-Flight Vehicle Atmospheric Environments, Natural Environments Branch/EV44, NASA/MSFC, Huntsville, AL 35812; ryan.k.decker@nasa.gov

${ }^{2}$ Natural Environments Engineer, Jacobs, Huntsville, AL 35806.
} 
during ascent. These assessments occur at predefined times during the launch countdown based on the most recent measured wind data. However, the pre-launch measured winds may not represent the wind environment during the vehicle ascent. Uncertainty in the UL winds over the time period between the assessment and launch can be mitigated by engineering analysis of critical vehicle trajectory variables and development of statistical models that provide the required level of protection. Trajectory allowances as a function of time interval that reduce the maximum allowable values ("knockdowns") of selected critical variables are made available during the countdown to ensure that the launch decision takes into account the uncertainty wind changes impose on trajectory assessments ${ }^{1-2}$. One method to quantify the distribution of

9 UL wind change at a launch site is to compile measured temporal wind profile pairs. ${ }^{3-5}$ In order to provide 10 robust engineering analyses the sample size must be large enough such that the sample population is 11 representative of a universal population at required probability levels. ${ }^{3,4}$ Historically, empirical statistical techniques of quantifying temporal UL wind change lacked sufficient sample sizes which required fitting 13 the empirical UL wind change distributions to theoretical extreme value probability distribution functions 14 in order to obtain estimates of UL wind change at extreme percentile levels. ${ }^{3}$ Over the past decade, 15 upgraded UL wind measurement systems have been operating at launch ranges and activities have been 16 conducted to archive UL wind data in order for use in vehicle trajectory assessments. ${ }^{6-7}$ Incorporating these 17 archived UL wind profiles will reduce the uncertainty and increase confidence in the quantification of empirical extreme UL wind change percentile levels for use in trajectory assessments and also reduce the 19 uncertainty in launch availability assessments.

20 Historical databases were collected from three locations: National Aeronautics and Space 21 Administration's (NASA's) Kennedy Space Center co-located on the United States Air Force's (USAF's) 22 Eastern Range (ER) at the Cape Canaveral Air Force Station, USAF's Western Range (WR) at Vandenberg 23 Air Force Base, and NASA's Wallops Flight Facility (WFF). These historical databases consist of a certain 24 number of wind pairs, where two wind profile measurements spaced by the time period of interest define a 25 pair. Sets of temporal wind pair databases for five time intervals $(0.75,1.5,2,3$, and 4 hours) were 26 generated from historical data at each location. 


\section{Data Sources}

Data from multiple UL wind measurement instrumentation systems were utilized to maximize sample size in the databases for each location. Wind profiles could either be from measurements by a rawinsonde balloon, a high-resolution Jimsphere balloon or multiple vertically pointing Doppler Radar Wind Profiler (DRWP) systems. The rawinsondes are lofted by latex balloons and transmit the data back to a groundbased receiving system. Rawinsondes typically reach $30.5 \mathrm{~km}$ (100 kft) before the balloon bursts. Output from rawinsonde data is usually presented in pressure levels, which corresponds to unevenly spaced altitude levels. To use rawinsonde data for vehicle response assessments, data are linearly interpolated to

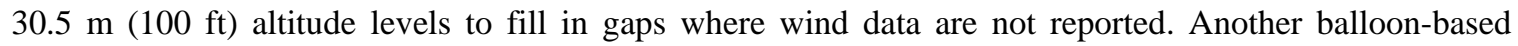

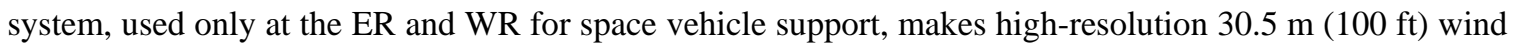
measurements through the use of a specially designed balloon known as a Jimsphere. ${ }^{8}$ There are two types of high-resolution wind measurement systems that can track Jimsphere ascent. One system uses groundbased radar to track a Mylar-coated Jimsphere balloon while another system, known as Real Time (RT) Automated Meteorological Profiling System (AMPS) High Resolution Flight Element (HRFE), uses Global Positioning Satellite technology and a clear Jimsphere balloon to track ascent. ${ }^{8-10}$ The AMPS system can also track ascent of a rawinsonde balloon, known as a Low Resolution Flight Element (LRFE). The Jimsphere balloon itself is more rigid than a rawinsonde, plus it contains roughness elements over the surface to disturb the air flow over the balloon and reduce the balloon's self-induced oscillation during ascent. ${ }^{8}$ The Jimsphere also contains a vent valve in order to maintain a constant volume and thus the balloon rises at a constant rate of $5 \mathrm{~m} / \mathrm{s}$ (10 knts). However, maintaining constant volume limits the altitude range the balloon can achieve. A Jimsphere can typically reach between 16.7-18.3 km (55-60 kft). ${ }^{8}$

Vertically pointing DRWP systems are ground-based instruments that transmit and receive electronic pulses that can be converted to wind speed and direction. At a minimum, two oblique beams pointing slightly off-zenith at orthogonal azimuth angles must exist to determine horizontal wind velocities, and a vertical beam must exist to determine vertical wind velocities. The DRWP transmitted frequency and antenna size dictates the altitude range sampled and the sampling interval. The ER has a 50-MHz and five 915-MHz DRWPs that, when their measurements are spliced together, can generate a wind profile from roughly $0.183-18.3 \mathrm{~km}(0.60-60 \mathrm{kft}){ }^{11}$ Data from the spliced profile are interpolated to a $15.2 \mathrm{~m}(50 \mathrm{ft})$ 
altitude interval. ${ }^{11}$. Unlike balloon-based systems, the DRWP operates continuously, with the $50-\mathrm{MHz}$ DRWP reporting measurements approximately every 5-mins and the 915-MHz DRWP reporting measurements approximately every 15-mins. Both DRWP systems produce wind profiles at vertical resolutions acceptable for launch vehicle assessments. These attributes yield orders of magnitude more profiles compared to balloon profiles available for developing temporal wind pair databases. The WR also has an equivalent set of DRWPs but due to time limitations data from both systems could not be integrated to generate vertically complete profiles.

\section{A. NASA Wallops Flight Facility}

Rawinsondes provide the only source of wind data at WFF, and two databases of rawinsonde profiles from WFF were obtained. The first was from the National Climatic Data Center (NCDC) Integrated Global

11 Radiosonde Archive (IGRA) ${ }^{12}$ database for the October 1963 through January 2000 period of record 12 (POR). IGRA consists of rawinsonde observations from sites around the globe and contains wind and 13 thermodynamic data at standard, surface and significant levels. The IGRA data for WFF consists of 14 balloons released from the National Weather Service (NWS) location near WFF. The other database of 15 rawinsondes was obtained directly from WFF, has a POR of February 2000 through January 2013, and 16 consists of rawinsondes released at the NWS site and at WFF in support of mission operations. The IGRA 17 database includes the rawinsonde data that was directly obtained from WFF personnel, which implies that no reason exists to include the IGRA data post December 1999 in the WFF wind pairs database generated.

\section{B. USAF Western Range}

Archived data from rawinsondes and Jimspheres were available for developing the WR wind pair databases. The data came from three sources: IGRA rawinsondes from January 1965 through January 2013, WR rawinsonde profiles from February 2008 through April 2012, and a Jimsphere database from January 1965 through September 2001. The time overlap between the IGRA and WR rawinsonde databases was necessary because the IGRA database contains only WR rawinsonde data. Whereas, the WR rawinsonde archive contains real-time AMPS (RTAMPS) LRFE wind data in addition to rawinsonde data that is archived in the IGRA database. As stated earlier, the WR wind pair databases do not contain DRWP measurements because the DRWP systems required extensive time to process. 


\section{USAF Eastern Range}

Data from the ER DRWP systems provide the largest sample size and are the sole source used for database development. The spliced ER DRWP profile database has a POR of April 2000 through December 2009. This POR results from the availability of quality controlled (QC'd) data for both the 50-MHz and 915-MHz DRWP. ${ }^{11}$ No rawinsonde or Jimsphere data were used because adding these data would have only increased the sample size of the ER database by $0.5 \%$.

8

$$
\text { Extensive QC of wind profile data were required to remove suspect data in individual profiles as well as }
$$
in profile pairs. Automated and manual QC checks were applied on the data from each measurement source. The automated QC checks differed between the measurement sources and consisted of general and study-specific checks. The latter checks were necessary because the general QC checks only evaluated data in single profiles and did not check consistency within a profile pair. A profile was rejected if it failed any given QC check. The following sections present details of the QC checks for each measurement system.

\section{A. Rawinsonde}

Rawinsonde data from all sources went through a two-step QC process. The rawinsonde data obtained from IGRA already were processed through a set of QC checks. ${ }^{12}$ An additional set of more stringent QC checks were performed on all rawinsonde data based on a manual review of the IGRA data. The QC checks assess data from an individual measurement perspective as well as at vertically adjacent levels. For a profile to be deemed acceptable for use, it must pass several QC checks. At least ten altitudes that contain either wind or thermodynamic data must exist. The minimum altitude in the profile must be positive. Also, all the heights reported must increase. For wind measurements the mean wind speed over the entire profile must be less than $51.4 \mathrm{~m} \mathrm{~s}^{-1}(100 \mathrm{kt})$. The vector differences between wind measurements at vertically adjacent levels must be less than $51.4 \mathrm{~m} \mathrm{~s}^{-1}$ (100 kt). A vertically adjacent check was also performed for ambient temperature where the difference magnitudes between vertically adjacent ambient temperature measurements in the lowest $3.048 \mathrm{~km}(10 \mathrm{kft})$ of the profile must be less than $20^{\circ} \mathrm{C}\left(68^{\circ} \mathrm{F}\right)$. Lastly the derived dew point temperature must correspond to ambient temperatures at least $-60^{\circ} \mathrm{C}\left(-76^{\circ} \mathrm{F}\right)$. 
The development process first extracted wind pairs containing profiles that passed all initial QC checks. However, a rigorous, iterative process applied several more QC checks on the data to ensure sufficient data quality within profile pairs. This process removed a wind pair that contained one or two profiles that failed a QC check. Wind data with each profile in the pair must reach a minimum of $\sim 6.1 \mathrm{~km}$ (20 kft). The minimum altitude requirement of $6.095 \mathrm{~km}$ (20 kft) was based on the minimum altitude required in order to perform certain launch vehicle assessments but insufficient for launches to altitudes of maximum aerodynamic pressure. A vertically adjacent check of wind component change was necessary since the rawinsonde data was linearly interpolated to fill in missing data that could result in artificially generated wind change. The wind change at vertically adjacent levels must not exceed $0.15 \mathrm{~s}^{-1}$. A related QC check was to reject profiles not containing at least $50 \%$ of the possible wind data. This check eliminated the potential of having an artificially large temporal wind change at the same altitude over the two profiles due to a large data interpolation in one of the profiles. A percentage check as opposed to an altitude range check was used due to the IGRA data being reported in pressure levels, which results in unequal altitude intervals and large data gaps inherently existing in valid profiles. The duplicate pair QC check was necessary for the WR wind profiles due to using multiple sources of wind profile databases with overlapping POR. If the profiles were less than or equal to 15 minutes apart they were grouped and the profile reaching the highest altitude was included in the database. Those unique profiles were then merged with the existing IGRA and RTAMPS database.

The last step entailed manually inspecting each pair. The development process implemented this step after a review of the maximum wind component change and probability distributions, independent of altitude, for each pair time interval. Temporal wind change analyses have shown that wind change extremes are typically correlated to time separation, where longer time intervals imply larger extreme wind change magnitudes. ${ }^{13}$ However, the WFF and WR wind component change at probability levels greater than 95\% in the 0.75 and 1.5 -hour pairs were $\sim 50 \%$ greater than the corresponding maximum wind change at the same probability level in the 2, 3 and 4-hour pairs without manual QC. Manual inspection of the WFF and WR wind pairs for all time intervals revealed these questionable wind change values were associated with profile pairs occurring around 0000 UTC in data obtained from the IGRA database. ${ }^{12}$ This characteristic appeared in the 2, 3 and 4-hour wind pairs for both WFF and WR. The differences observed in the 
questionable wind profiles seemed more characteristic of diurnal-scale wind change as opposed to shorttime period wind change, which led to questioning the time stamp of each profile in the pair. An

3 independent source provided a comparison to events of questionable wind change. Data from the National

4 Centers for Environmental Prediction (NCEP) North American Regional Reanalysis project at the time 5 period of interest were evaluated to determine if a large gradient in the winds existed over the time period.

6 The majority of these cases did not corroborate with the NCEP data and, as a result, the wind pairs 7 occurring near 0000 UTC that contained wind profiles from the IGRA source were removed from the 8 generated database. The resultant temporal wind change distributions were better correlated as a function of 9 time separation.

\section{B. Jimsphere}

The WR wind pairs include wind profiles from both systems that use the Jimsphere balloon. Jimsphere wind profiles were generated during launch vehicle operations and were manually QC'd by technicians

13 prior to distribution to launch vehicle operators. The manual QC checks were performed to remove suspect 14 data for use in flight vehicle assessments. ${ }^{6}$ A profile was removed if its lowest altitude exceeded $122.2 \mathrm{~m}$ 15 (400 ft) or if the profile contained any decreasing altitudes. All variables (altitude, wind speed, and wind 16 direction) were removed at a specific altitude if at least one variable were missing and the remaining data 17 above were removed after the first altitude containing missing data. Any linear wind component interpolations at the top of the profile were also removed.

19 The selected wind pairs for the WR can be made up of two Jimspheres, two rawinsondes, or a 20 Jimsphere and a rawinsonde. The issue with the Jimsphere/rawinsonde combination is that a difference 21 exists in the smallest resolvable wavelengths between these two wind profiles due to their sampling 22 intervals. The small-scale wavelengths were removed through a filtering algorithm in order to maintain an 23 equivalent effective vertical resolution between the rawinsonde and Jimsphere systems. ${ }^{8}$ A $244.3 \mathrm{~m}$ (800 ft) 24 filter was applied to the Jimsphere based on a power spectrum analysis of the rawinsonde data shown in 25 Fig. 1. Filtering the Jimsphere data was necessary to use wind profiles from either system interchangeably 26 in assessing wind effects on vehicle performance. ${ }^{8}$ The RTAMPS mean normalized power spectral density 27 likely contains additional noise in the 152-610 m (500-2000 ft) wavelength range. The additional noise was 28 not removed from the RTAMPS data contained in the database since filtering the Jimsphere data to $\sim 610 \mathrm{~m}$ 
( $2000 \mathrm{ft}$ ) would remove valid spectral content that is necessary to assess wind affects on vehicle

2 performance.

3 C. DRWP

The ER wind pairs consist of profiles from the DRWP systems, which are designed to operate continuously with limited manual QC processing. Various algorithms and methodologies were applied in the QC process of the 50-MHz DRWP (D-50) data and in the 915-MHz DRWP (D-915) automated QC process. ${ }^{7,14}$ Additional QC on the D-915 data included removing profiles with duplicate timestamps, filling temporal data gaps greater than 15 minutes, and checking for correct altitude progression. The resultant POR of temporal overlapping QC'd D-50 and D-915 data extends from April 2000 to December 2009.

The next step after data QC entailed creating a single profile by splicing the two DRWP profiles at the altitude where the top of the D-915 profile, 6,100 m (20,013 ft) at most, and the bottom of the D-50 profile, approximately 2,500 $\mathrm{m}(8,202 \mathrm{ft})$, meet or overlap. Before generating the spliced DRWP profiles, the temporal and spatial (vertical) criteria are determined and applied to all DRWP profiles because the individual profiles had to match in both domains before splicing. ${ }^{11}$ The D-50 archive contains measurements at $150 \mathrm{~m}$ (492 ft) intervals every five minutes prior to an instrument upgrade in 2004 and

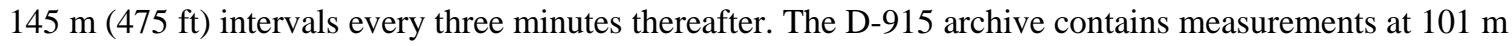
(331 ft) intervals roughly every 15 minutes. The spatial criterion for the blended profiles is $50 \mathrm{~m} \mathrm{(164 \textrm {ft } )}$ with the temporal criterion based on the time interval of the D-50 measurements. each of the D-915 profiles. Next, the algorithm interpolated the D-50 and D-915 profiles to $50 \mathrm{~m}$ (164 ft) spacing in the altitude range $0.100-18.6 \mathrm{~km}$ (328-61,024 ft), which corresponds to the lowest observation of the D-915s and the highest observation of the D-50. Then, the algorithm counted the number of data gaps from each instrument, flagged $300 \mathrm{~m}(984 \mathrm{ft})$ data gaps with the D-50 and $500 \mathrm{~m}$ (1640 ft) data gaps with the D-915, and linearly interpolated wind components within un-flagged gaps. Both profiles contained data placeholders at the same altitudes, with a transition region existing between the two profiles typically at altitudes around 2,000-3,000 $\mathrm{m}(6,562-9,843 \mathrm{ft})$. The algorithm then spliced the two profiles using a methodology that varied slightly based on the data coverage of the two profiles within the transition region. 
If a D-915 profile overlapped the D-50 profile, then the algorithm combined the D-50 and D-915 wind components within the transition region using a weighting scheme that provided greater weight to the D-915 at lower altitudes and greater weight to the D-50 at higher altitudes. If a D-915 profile did not reach the D-50 profile, then the algorithm linearly interpolated the winds between the highest altitude of the D915 profile and the lowest altitude of the D-50 profile, provided the QC algorithm did not flag the gap.

6 Each spliced profile contained winds exclusive to the D-915 below the transition region, derived winds within the transition region, and winds exclusive to the D-50 above the transition region. Splicing the D-50 and individual D-915 profiles produced up to five DRWP wind profiles at a given timestamp in the archive, 9 depending on the QC process and how many D-915s were operating.

The individual spliced profiles were then combined to generate a single composite DRWP profile 11 representing the wind environment at a given timestamp. The individual spliced profiles only differed 12 below the lowest altitude of the D-50 profile. An algorithm was developed that generated composite winds 13 at each altitude starting at $150 \mathrm{~m}(492 \mathrm{ft})$ and ending at 18,450 m (60,532 ft). The algorithm omitted data at 14 the lowest D-915 altitude and the highest D-50 altitude due to ground clutter effects and questionable 15 shears, respectively. The algorithm first computed a mean reference wind using the individual spliced 16 profiles with valid winds at each altitude. Next, vector differences between the winds from each of the 17 individual spliced profiles and the reference wind were used to derive weights corresponding to each of the 18 profiles. Summing the product of the weights and wind components from all individual profiles produced

19 the composite wind at each altitude. Above the lowest reporting altitude, the algorithm computed the 20 reference wind as the mean of the reference wind described above and the composite wind at the previous 21 altitude.

22 A subsample of the DRWP archive was produced according to specified guidelines. The wind profile 23 had to contain data at all altitudes from 250-6,096 m (820-20,000 ft) and the wind components were 24 linearly reduced from altitudes below the lowest reporting altitude, which ranged from 150-250 m (492-820 $25 \mathrm{ft})$, to no wind at $0 \mathrm{~m}(0 \mathrm{ft})$. Profile tops extended as high as 18,450 $\mathrm{m}(60,532 \mathrm{ft})$. 


\section{Wind Pair Development and Analysis}

The criteria to select pairs, the resultant number of wind pairs at each location, statistical analyses of the sample sizes, and distributions of extreme wind changes are presented in the following sections.

\section{A. Criteria to select pairs}

Constraining the pair selection to the exact time spacing with the balloon-based WFF and WR profiles limits the number of pairs since balloons are released infrequently. Therefore, for each pair the time range was expanded by +/- 15 minutes to increase the wind pair sample size. For example, profiles spaced between 2.75 to 3.25 hours were treated as 3-hour pairs. For the ER, two profiles defined a pair if the desired time separation of the pair +/- two minutes separated the profiles' timestamps. For example, a 0.75hour (45-minute) pair has two profiles spaced anywhere from 43-47 minutes apart. The pair selection process used a two-minute window because a large number of DRWP profiles existed and at least three minutes existed between adjacent DRWP profiles.

\section{B. Sample Size}

Table 1 presents the number of pairs at each time interval and location. Depending on the time interval of interest, the WFF, WR, and ER databases contain 54-127 pairs, 366-548 pairs, and 260,878297,490 pairs, respectively. The disparity in the magnitude of samples at the ER is due to the continuous operation of the DRWP versus the discrete measurements from the balloon systems used at WR and WFF. The WR's history of supporting space launch operations that require frequent balloon releases attributes to the difference between the number of WR and WFF pairs.

\section{Statistical Analysis}

The most frequent application of wind pair databases is to apply the empirical maximum zonal (u) and meridional (v) wind change components of each profile into a persistence assessment to determine the effects of wind change over a specific time period on vehicle performance. ${ }^{5}$ Therefore, a large sample size must exist in order to capture the largest range of maximum wind change possible. Several analyses were conducted to determine how well the sample population at each location characterized the wind change extremes.

The analyses results quantify the distribution and the confidence bound (CB) in the empirical maximum wind change from the various sample sizes of each pair set. Extreme wind change population 
distributions are usually non-Gaussian ${ }^{15}$, so an extreme theoretical probability function was used to fit the data. The generalized extreme value (GEV) probability distribution function (PDF) ${ }^{16-17}$ provided a good fit of the extreme u- and v-component wind changes in each pair up to roughly the 99th percentile level. The GEV PDF is expressed by:

$$
y=f(x \mid k, \mu, \sigma)=\left(\frac{1}{\sigma}\right) \exp \left\{-\left[1+k \frac{(x-\mu)}{\sigma}\right]^{-\frac{1}{k}}\right\}\left[1+k \frac{(x-\mu)}{\sigma}\right]^{-1-\frac{1}{k}}
$$

$$
\text { for } k \neq 0 \text { and } 1+k \frac{(x-\mu)}{\sigma}>0
$$
where $x$ represents each value in a distribution of wind changes, and $k, \mu$, and $\sigma$ denote the scale, shape, and location parameters, respectively, of the GEV estimate. Using the results from the GEV, 95\% CB at various percentile levels were calculated using the Asymptotic Distribution of Percentiles (ADP) method. ${ }^{18}$ The

10 ADP equation is a function of the CB, sample size and percentile level of interest. The analysis uses the $1195 \%$ CB as a conservative approach to assess the range of extreme wind change for selected percentile 12 levels.

13 Representing the data annually in the distribution plots for all sites was necessary due to the limited 14 sample populations from WR and WFF. Seasonal and monthly variability does exist in wind component 15 distributions. ${ }^{13}$ When using the data for engineering assessments, ideally, data should be grouped by season 16 or month to ensure the "knockdown" value is based on a homogenous sample population. However, a 17 database subset representing a given month or season must contain a sample size large enough to characterize the environment to the desired probability level. The ER database, which contains enough 19 samples for monthly assessments, was compared to the 2013 Cape Canaveral Air Force Station Range Reference Atmosphere (RRA). ${ }^{19}$ The RRA contains statistics of wind variables derived from rawinsonde balloon measurements at the ER. Statistics are reported as a function of altitude for each month and 22 annually. The comparison first computed the statistics listed in the RRA from the ER database, and then overlaid results from both sources. The annual results compared very well with some minor differences for an individual month as shown in Fig. 2. These differences are likely caused by the different sampling 25 characteristics between the DRWP systems and the balloon-based systems that were used to derive the 26 RRA. 
Annual distribution plots of the maximum change in wind component magnitude are presented in Figures 3-6 to illustrate differences in the $\mathrm{CB}$ as a function of sample population. For each plot, the cumulative probability, drawn from the probability density function ${ }^{20}$, is along the $y$-axis and the magnitude of the wind component's change is along the x-axis. The sample size of the pairs is correlated to the width of uncertainty at the 95\% CB for the highest percentile levels in the sample population. As the sample size decreases the width of uncertainty at the 95\% CB increases. Figures 3 and 5 show the variation in the width of uncertainty at the 95\% CB. In addition, a small probability density at higher percentile levels increases the width of uncertainty. Figure 3, the WFF 2-hour pairs plot, shows this attribute - where the 95\% CB at probability levels above $95 \%$ in the v-component change significantly exceeds the width of the CB in the corresponding u-component change even though the sample sizes for both u- and v-changes are the same.

The width of uncertainty in the CB for all the ER pairs is small due to the large sample size. An example of an ER pair fit to the CB is shown in Fig. 4. The deviation of the CB from the empirical distribution above the 95th percentile level is an artifact of the CB being calculated from the GEV function, which does not fit the empirical distribution well. However, the poor fit is not an issue since the sample size is large enough to justify using the empirical percentiles for almost any flight vehicle assessment. size decreases. The WR 95\% CB range of uncertainty, as shown in Table 2, at the sample size's maximum empirical probability level was approximately $15.4 \mathrm{~m} \mathrm{~s}^{-1}$ (30 kt) for both wind components in all the pairs with the exception for the 4-hour pairs. The range of uncertainty at the maximum empirical probability level for the 4-hour pairs is $\sim 40.1 \mathrm{~m} \mathrm{~s}^{-1}$ (78 kt) as shown in Fig. 5. Because of the large uncertainty at the extreme empirical percentile in the 4-hour pairs, another approach was applied to quantify the confidence of the empirical wind change data. This approach uses a function that approximates the probability level of a sample population with a specified sample size to a probability level of the universal population. ${ }^{3}$ The function makes no assumption to the form of the probability distribution function of the wind change and is defined as: where $P_{u}$ is the probability that the sample contains the universal population at the sample probability $P_{s}$ 
sample contains the $P_{s}$ value of the universal population. Table 3 presents the confidence level of the universal population for various sample probability levels based on the sample size in each WR wind pair interval. For the 366 4-hour wind pairs, there is $88.1 \%$ confidence that the pairs contain the 99th percentile of wind change during this time interval. The confidence level exceeds $90 \%$ for the other four time periods. These samples are large enough for most vehicle performance applications; however, a low confidence exists that these samples capture wind changes at extreme (e.g., > 99th percentile) levels.

The WFF samples contain the smallest number of pairs of the three locations. Due to the small sample sizes for each time period, the 95\% CB for the observed wind change extremes have a large uncertainty. The range of the 95\% CB for the v-component wind change range is at least $20.6 \mathrm{~m} \mathrm{~s}^{-1}$ (40 kt) for each time period. The observed maximum 4-hour v-component wind change of $37.0 \mathrm{~m} \mathrm{~s}^{-1}$ (74 kt) corresponds to a 98th percentile level in the sample population's distribution. However, the 95\% CB at the 98th percentile level ranges from $\sim 40$ to $89 \mathrm{kt}\left(20.6\right.$ to $45.8 \mathrm{~m} \mathrm{~s}^{-1}$ ) as shown in Fig. 6.

Table 4 presents confidence levels of the universal population for various sample probabilities based on the WFF sample size. A $16.9 \%$ confidence exists that the 4-hour pairs contain the 99th percentile of all wind changes during this period. The confidence levels range from $10-36 \%$ at the 99th percentile for the other pairs. Due to the low confidence that the sample contains extreme wind changes and large uncertainty in the 95th confidence intervals at probability levels above 95\%, the recommendation is to apply the extreme 4-hour wind component change for all time change intervals of interest in vehicle performance evaluations. Applying this recommendation produces more conservative results for shorter time periods, while increasing the likelihood of under-conservative results as the time period approaches 4-hours.

Table 5 presents confidence levels of the universal population for various sample probabilities based on the ER sample size. The confidence level is $100 \%$ for all time periods.

\section{Conclusion}

Temporal upper level wind pair databases were generated for incorporation into commit-to-launch decisions based on upper level wind assessments. Databases for five time intervals $(0.75,1.5,2,3$, and 4 hours) at the National Aeronautics and Space Administration's Kennedy Space Center co-located on the United States Air Force's Eastern Range at the Cape Canaveral Air Force Station, the United States Air 
1 Force's Western Range at Vandenberg Air Force Base, and National Aeronautics and Space

2 Administration's Wallops Flight Facility were generated through use of historical data at each location.

3 Multiple sources that measure upper level atmospheric winds at the requested sites were used for this study.

4 Databases were compiled using wind profiles from rawinsonde, Jimsphere, and Doppler Radar Wind

5 Profiler systems. Extensive quality control checks were applied on the data to remove unacceptable

6 profiles. Statistical analyses of the resultant wind pairs from each site were performed to determine if the

7 observed extreme wind changes in the sample pairs represent extreme temporal wind change. The resultant

8 Eastern Range wind pair databases yielded sample sizes that characterize the extreme wind change

9 environment and no restrictions on the usage exist. The Western Range wind pair database sample size is

10 large enough for vehicle performance assessments up to the 99th percentile level. Including data from the

11 Western Range Doppler Radar Wind Profiler systems would increase the sample size of temporal wind pair

12 databases in an effort to characterize probability levels above the 99th percentile level. However, The

13 Wallops Flight Facility database contains too small of a sample size to characterize extreme wind changes.

14 This characteristic yields to low confidence existing that the observed extremes in each time period

15 characterizes the extreme wind change environment. Therefore, for any vehicle performance applications at

16 Wallops Flight Facility, the recommendation is to either apply the extreme 4-hour wind change values for

17 all time change intervals of interest or incorporate analytical statistical techniques to fit the empirical

18 distribution in order to characterize extreme wind change events.

\section{Acknowledgements}

Thanks to NASA's Launch Services Program office who sponsored the study, staff at NASA's Applied Meteorology Unit who provided technical review and members of the MSFC Natural Environments Branch for the technical discussions on wind statistics and spectral analysis. Mr. Tyler Brock from USAF provided the Vandenberg Air Force Base rawinsonde data. Mr. Brian Cunningham from LIT and Associated, Inc. provided the Wallops Flight Facility rawinsonde data and answered questions relating to timestamp discrepancies. Mr. Matt McClelland from United Launch Alliance provided additional Vandenberg Air Force Base Jimsphere and rawindsonde data. Dr. Brian Sako from Aerospace Corp. provided additional Vandenberg Air Force Base Jimsphere data. 


\section{References}

${ }^{1}$ Smith, O. E. and Austin L. D., “Space Shuttle Response to Ascent Wind Profiles," Journal of Guidance, Control and Dynamics, Vol. 6, No. 5, 1983, pp. 355-360.

${ }^{2}$ Smith, O. E. and Adelfang, S. I., "Wind Profile Models: Past, Present and Future for Aerospace Vehicle Ascent Design,” AIAA Paper 98-1047, January, 1998.

${ }^{3}$ Smith, O. E. and Adelfang, S. I., "A Compendium of Wind Statistics and Models for the NASA Space Shuttle and Other Aerospace Vehicle Programs,” NASA/CR-1998-208859, October, 1998.

${ }^{4}$ Wilfong, T. L., Creasey, R. L., and Smith, S. A., "Wind Persistence from 2-18 km Using a Wind Profiler,” AIAA Paper 93-0854, January, 1993.

${ }^{5}$ Smith, O. E. and Adelfang, S. I., “STS Ascent Structural Loads Statistics,” AIAA Paper 92-0720, January, 1992.

${ }^{6}$ Divers, R., Viens, P., Mitchell, T., Bzdusek, K., Herman, G., Hoover, R., “Automated Meteorological Profiling System (AMPS) Description” American Meteorological Society, Paper J8.1, September 2000.

${ }^{7}$ Barbré, R. E., “Quality control algorithms for the Kennedy Space Center 50-MHz Doppler radar wind profiler winds database,” Journal of Atmospheric and Oceanic Technology, December 2012.

${ }^{8}$ Wilfong, T. L., Smith, S. A., and Crosiar C. L., "Characteristics of High-Resolution Wind Profiles Derived from Radar-Tracked Jimspheres and the Rose Processing Program," Journal Atmospheric and. Oceanic Technology, April 1997.

${ }^{9}$ Adelfang, S. I., “Analysis of Near Simultaneous Jimsphere and AMPS High Resolution Wind Profiles,” AIAA Paper 2003-895, Jan. 2003.

${ }^{10}$ Wilfong, T. L., M. L. Maier, C. L. Crosiar, M.S. Hinson and B. Divers, "Characteristics of Wind Profiles Derived from GPS Based Automated Meteorological Profiling System (AMPS)” American Meteorological Society, Paper J8.2, September 2000.

${ }^{11}$ Barbré, R. E., "Characteristics of the Spliced KSC Doppler Radar Wind Profiler Database”. Jacobs ESSSA Group Analysis Report, ESSSA-FY13-1935, Huntsville, AL, November 2013.

${ }^{12}$ Durre, I., Vose R. S., and Wuertz D. B., “Overview of the Integrated Global Radiosonde Archive,” Journal of Climate, January 2006.

${ }^{13}$ Johnson, D. L., “Terrestrial Environment (Climatic) Criteria Handbook for use in Aerospace Vehicle Development,” NASA-HDBK-1001, August 2000.

${ }^{14}$ Lambert, W. C., Merceret F. J., Taylor G. E., and Ward J. G., "Performance of five 915-MHz wind profilers and an associated automated quality control algorithm in and operational environment," Journal of Atmospheric and Oceanic Technology, November 2003.

${ }^{15}$ Merceret, F. J., "Rapid temporal changes of midtropospheric winds," Journal of Applied Meteorology, November 1997.

${ }^{16}$ Coles, S., An Introduction to Statistical Modeling of Extreme Values, Springer Series in Statistics, London, 2001.

${ }^{17}$ Kotz, S., and Nadarajah S., Extreme Value Distributions: Theory and Applications, Imperial College Press, London, 2000.

${ }^{18}$ DasGupta, A., Asymptotic Theory of Statistics and Probability, Springer Texts in Statistics, London, 2008.

${ }^{19}$ Burns, K.L., "Range Reference Atmosphere 2013: Cape Canaveral Air Force Station, Florida”. Jacobs ESSSA Group Analysis Report, ESSSA-FY13-1655, Huntsville, AL, December 2013.

${ }^{20}$ Wilks, D. S., Statistical Methods in the Atmospheric Sciences. 2d ed., Academic Press, San Diego, 2006. . 
Table 1 Sample size of wind pairs at each location.

\begin{tabular}{|c|c|c|c|}
\hline $\begin{array}{c}\text { Time Interval } \\
\text { (hours) }\end{array}$ & ER & WR & WFF \\
\hline 0.75 & 273,265 & 435 & 78 \\
\hline 1.5 & 260,878 & 401 & 54 \\
\hline 2 & 297,490 & 548 & 75 \\
\hline 3 & 273,189 & 508 & 127 \\
\hline 4 & 276,108 & 366 & 74 \\
\hline TOTAL & $1,380,930$ & 2258 & 408 \\
\hline
\end{tabular}

Table 2 The WR 95\% CB range of uncertainty for u- and v-wind component wind change at each pair's maximum empirical probability level.

\begin{tabular}{|c|c|c|}
\hline $\begin{array}{c}\text { Time Interval } \\
\text { (hours) }\end{array}$ & $\begin{array}{c}\text { u-component wind } \\
\text { change (knts) }\end{array}$ & $\begin{array}{c}\text { v-component wind } \\
\text { change (knts) }\end{array}$ \\
\hline 0.75 & 28.47 & 20.54 \\
\hline 1.5 & 23.50 & 24.17 \\
\hline 2 & 24.24 & 23.30 \\
\hline 3 & 30.44 & 34.47 \\
\hline 4 & 78.25 & 75.34 \\
\hline
\end{tabular}


1

11

Table 3 Confidence levels of the universal population for arbitrarily selected sample probability levels and the WR sample size for each wind pair time interval (Smith and Adelfang 1998).

\begin{tabular}{|c|c|c|c|c|c|}
\hline \multirow{2}{*}{$\begin{array}{c}\text { Sample } \\
\text { Probability }\end{array}$} & \multicolumn{5}{|c|}{ Time Interval (Sample Size) } \\
\cline { 2 - 6 } & 0.75 hours & 1.5 hours & 2 hours & 3 hours & 4 hours \\
$(435)$ & $(401)$ & $(548)$ & $1.08)$ & $(366)$ \\
\hline 0.500 & 1.0000 & 1.0000 & 1.0000 & 1.0000 & 1.0000 \\
\hline 0.750 & 1.0000 & 1.0000 & 1.0000 & 1.0000 & 1.0000 \\
\hline 0.900 & 1.0000 & 1.0000 & 1.0000 & 1.0000 & 1.0000 \\
\hline 0.950 & 1.0000 & 1.0000 & 1.0000 & 1.0000 & 1.0000 \\
\hline 0.990 & 0.9318 & 0.9102 & 0.9734 & 0.9628 & 0.8813 \\
\hline 0.995 & 0.6400 & 0.5960 & 0.7592 & 0.7215 & 0.5466 \\
\hline 0.999 & 0.0711 & 0.0617 & 0.1050 & 0.0925 & 0.0525 \\
\hline
\end{tabular}

Table 4 Confidence levels of the universal population for arbitrarily selected sample probability levels and the WFF sample size for each wind pair time interval (Smith and Adelfang 1998).

\begin{tabular}{|c|c|c|c|c|c|}
\hline \multirow{2}{*}{$\begin{array}{c}\text { Sample } \\
\text { Probability }\end{array}$} & \multicolumn{5}{|c|}{ Time Interval (Sample Size) } \\
\cline { 2 - 6 } & $\begin{array}{c}0.75 \text { hours } \\
(78)\end{array}$ & $\begin{array}{c}1.5 \text { hours } \\
(54)\end{array}$ & $\begin{array}{c}2 \text { hours } \\
(75)\end{array}$ & $\begin{array}{c}3 \text { hours } \\
(127)\end{array}$ & $\begin{array}{c}\text { 4 hours } \\
(74)\end{array}$ \\
\hline 0.500 & 1.0000 & 1.0000 & 1.0000 & 1.0000 & 1.0000 \\
\hline 0.750 & 1.0000 & 1.0000 & 1.0000 & 1.0000 & 1.0000 \\
\hline 0.900 & 0.9973 & 0.9763 & 0.9965 & 0.9999 & 0.9962 \\
\hline 0.950 & 0.9065 & 0.7592 & 0.8944 & 0.9886 & 0.8900 \\
\hline 0.990 & 0.1836 & 0.1018 & 0.1729 & 0.3629 & 0.1693 \\
\hline 0.995 & 0.0584 & 0.0301 & 0.0545 & 0.1332 & 0.0532 \\
\hline 0.999 & 0.0028 & 0.0013 & 0.0026 & 0.0073 & 0.0025 \\
\hline
\end{tabular}


1

2

3

4 5

7

8

9

10
Table 5 Confidence levels of the universal population for arbitrarily selected sample probability levels and the ER sample size for each wind pair time interval (Smith and Adelfang 1998).

\begin{tabular}{|c|c|c|c|c|c|}
\hline \multirow[b]{2}{*}{$\begin{array}{c}\text { Sample } \\
\text { Probability }\end{array}$} & \multicolumn{5}{|c|}{ Time Interval (Sample Size) } \\
\hline & $\begin{array}{c}0.75 \text { hours } \\
(273,265)\end{array}$ & $\begin{array}{l}1.5 \text { hours } \\
(260,878)\end{array}$ & $\begin{array}{c}2 \text { hours } \\
(297,490)\end{array}$ & $\begin{array}{c}3 \text { hours } \\
(273,189)\end{array}$ & $\begin{array}{c}4 \text { hours } \\
(276,108)\end{array}$ \\
\hline 0.500 & 1 & 1 & 1 & 1 & 1 \\
\hline 0.750 & 1 & 1 & 1 & 1 & 1 \\
\hline 0.900 & 1 & 1 & 1 & 1 & 1 \\
\hline 0.950 & 1 & 1 & 1 & 1 & 1 \\
\hline 0.990 & 1 & 1 & 1 & 1 & 1 \\
\hline 0.995 & 1 & 1 & 1 & 1 & 1 \\
\hline 0.999 & 1 & 1 & 1 & 1 & 1 \\
\hline
\end{tabular}




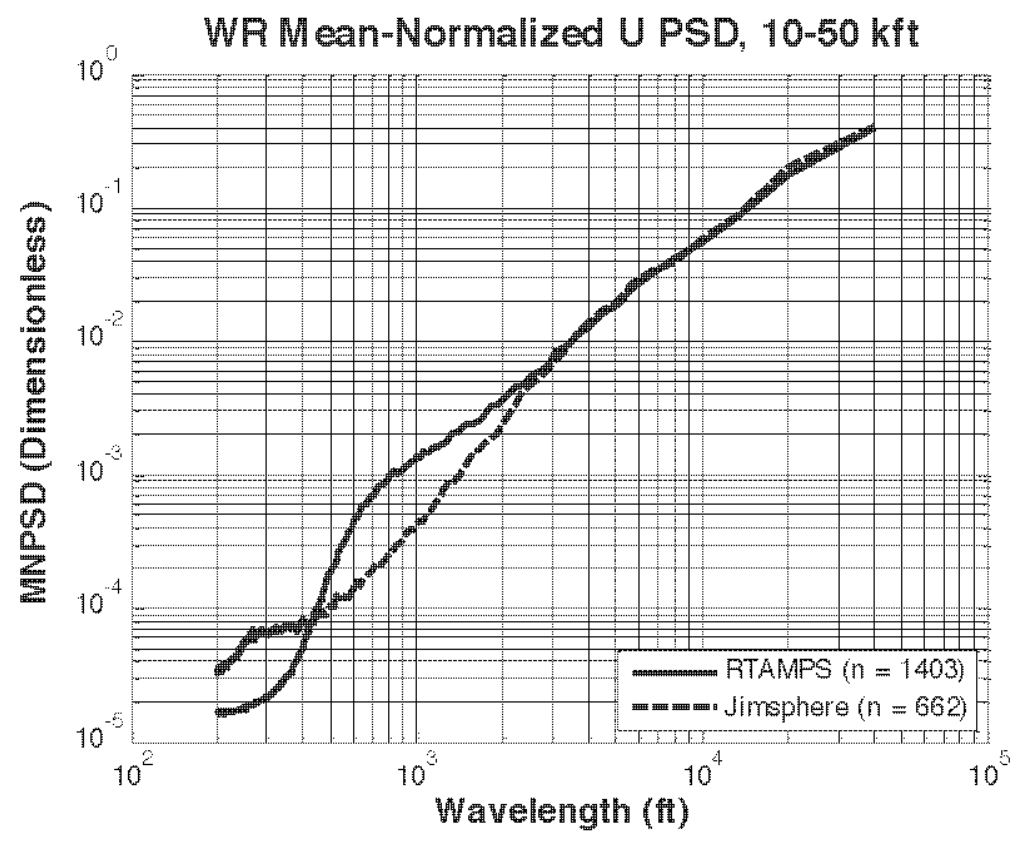

1

a)

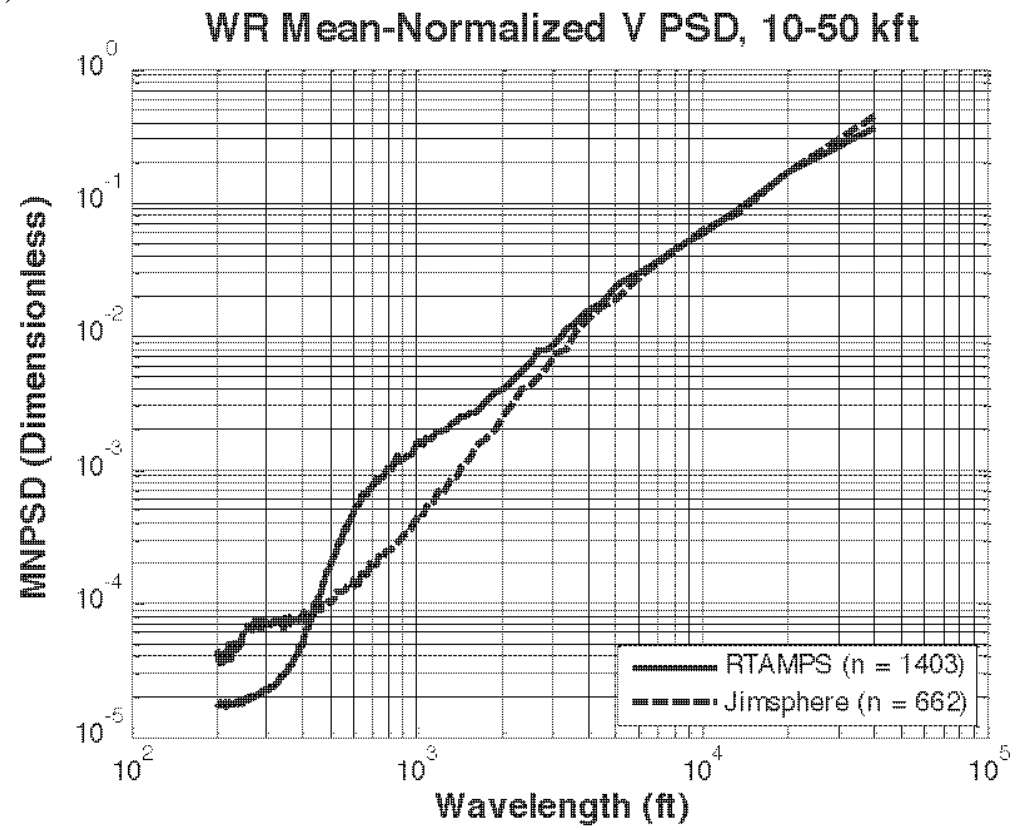

b)

Fig 1. Mean-normalized power spectral density (PSD) for the u-component (a) and v-component (b) WR Jimsphere and RTAMPS rawinsonde systems. 

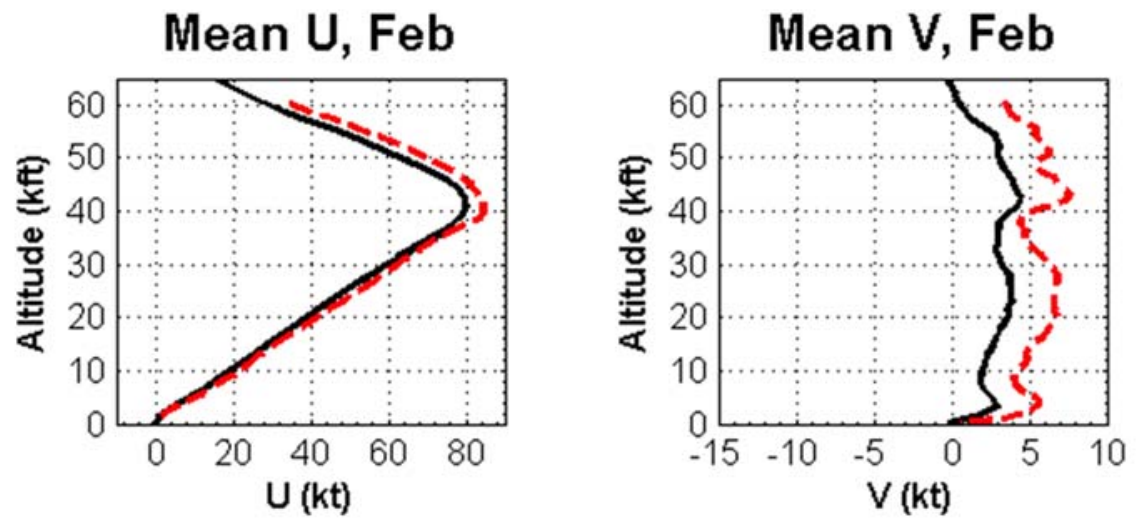

a)
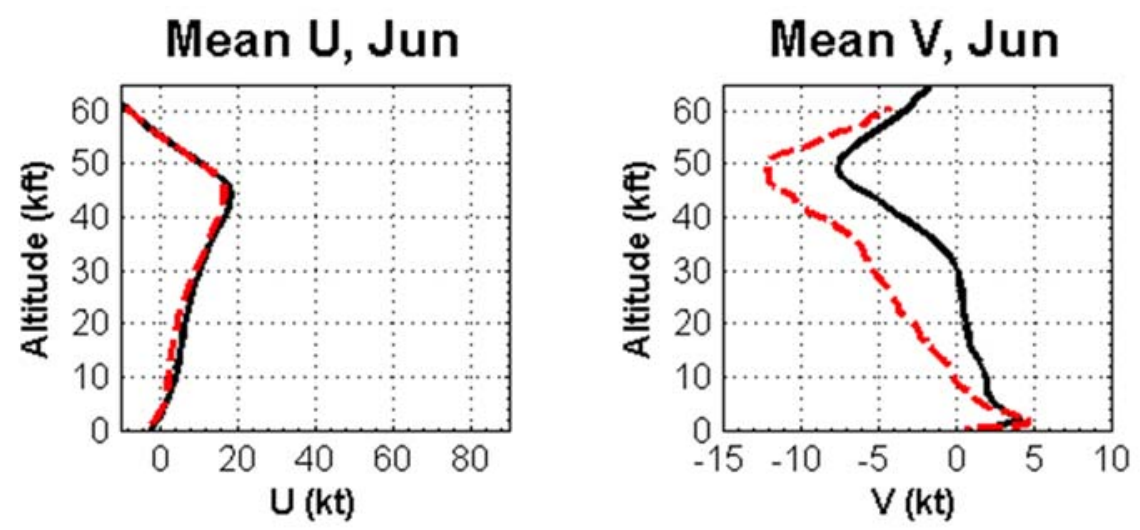

b)
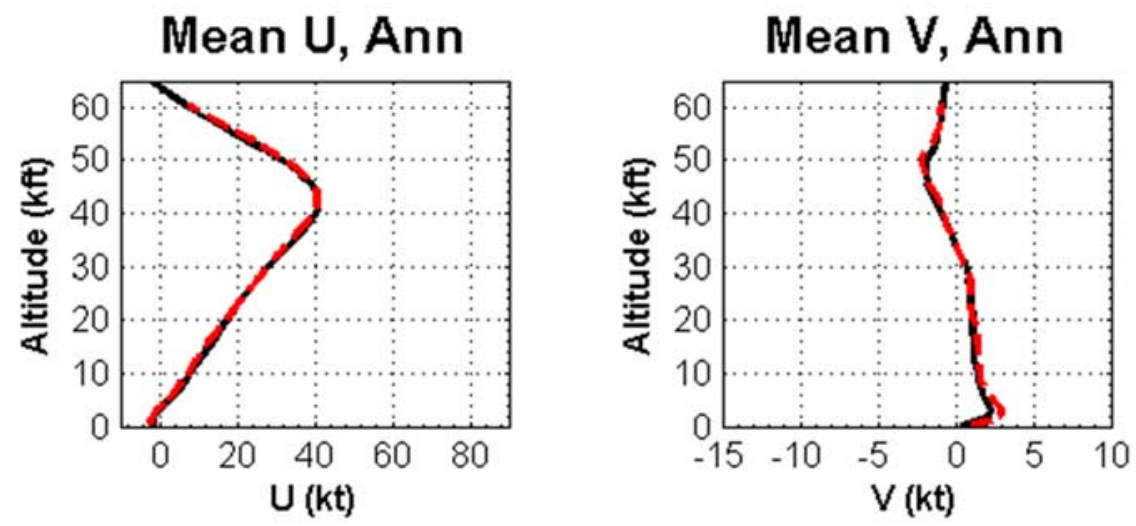

c)

Fig 2. Comparison of ER DRWP profiles contained in 4-hr pairs database with the 2013 CCAFS RRA database statistics for February (a) monthly mean u- and v-component (solid line - CCAFS RRA; dashed line ER DRWP), June (b) monthly mean u- and v-component (solid line - CCAFS RRA; dashed line ER DRWP) and Annual (c) mean u- and v-component (solid line - CCAFS RRA; dashed line ER DRWP). 


\section{WFF Maximum 2-hr u-component Change Magnitudes with Generalized Extreme Value Fit and $95 \%$ Confidence Bounds, $\mathrm{n}=75$}

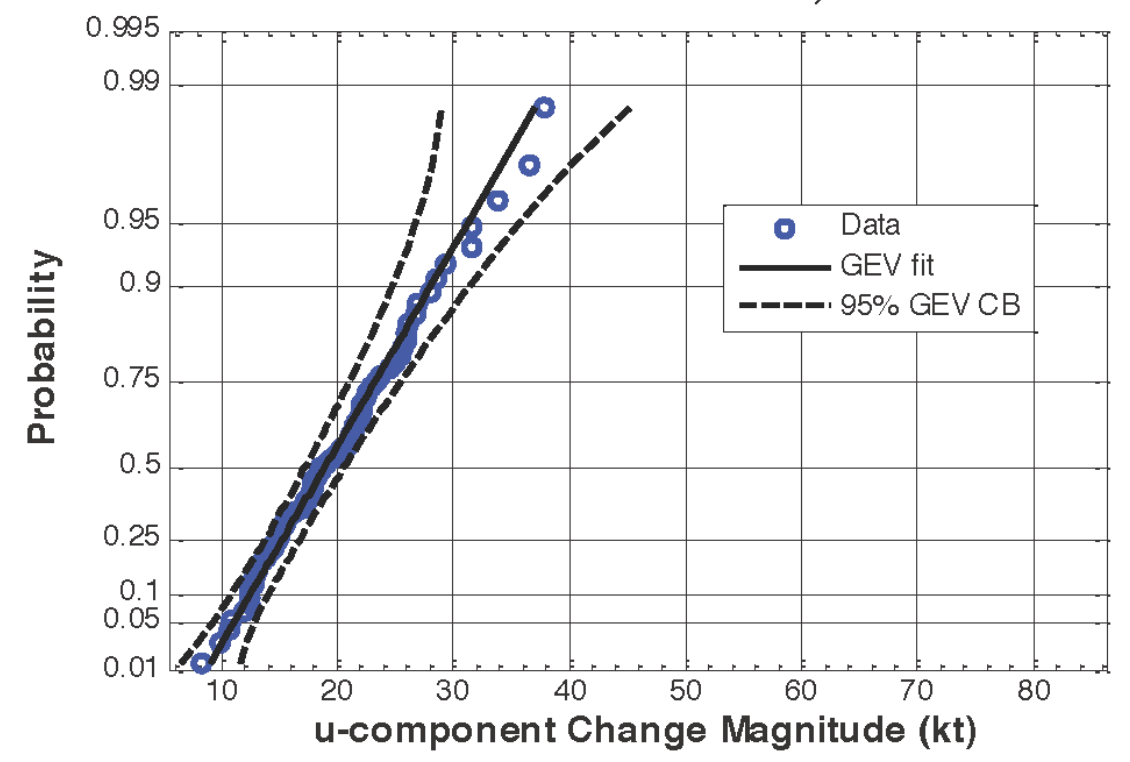

a)

WFF Maximum 2-hr v-component Change Magnitudes with Generalized Extreme Value Fit and $95 \%$ Confidence Bounds, $\mathrm{n}=75$

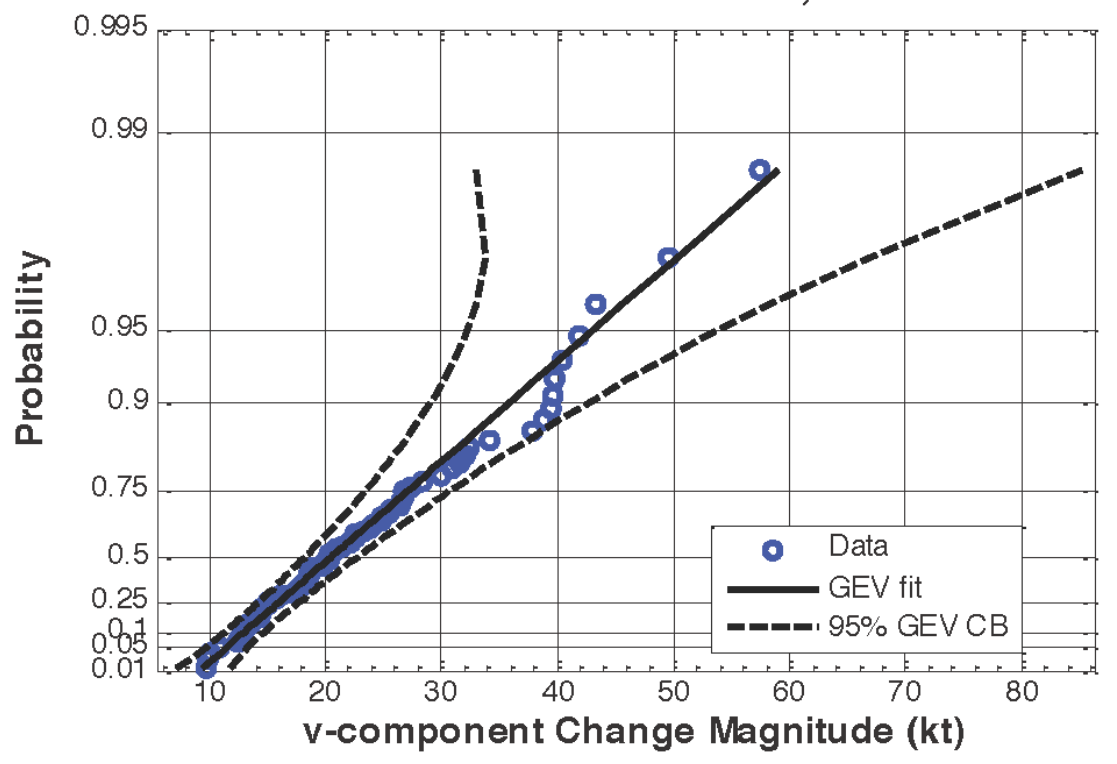

b)

Fig 3. WFF maximum wind change from the 2-hour wind pairs with $95 \%$ CB for the $u-(a)$ and vcomponent (b) wind changes. The magnitude of the wind component change is on the $x$-axis and probability is on the y-axis. The number (n) of pairs in the analysis is 75. 
ER Maximum 4-hr u-component Change Magnitudes with Generalized Extreme Value Fit and 95\% Confidence Bounds, $\mathrm{n}=\mathbf{2 7 6 1 0 8}$

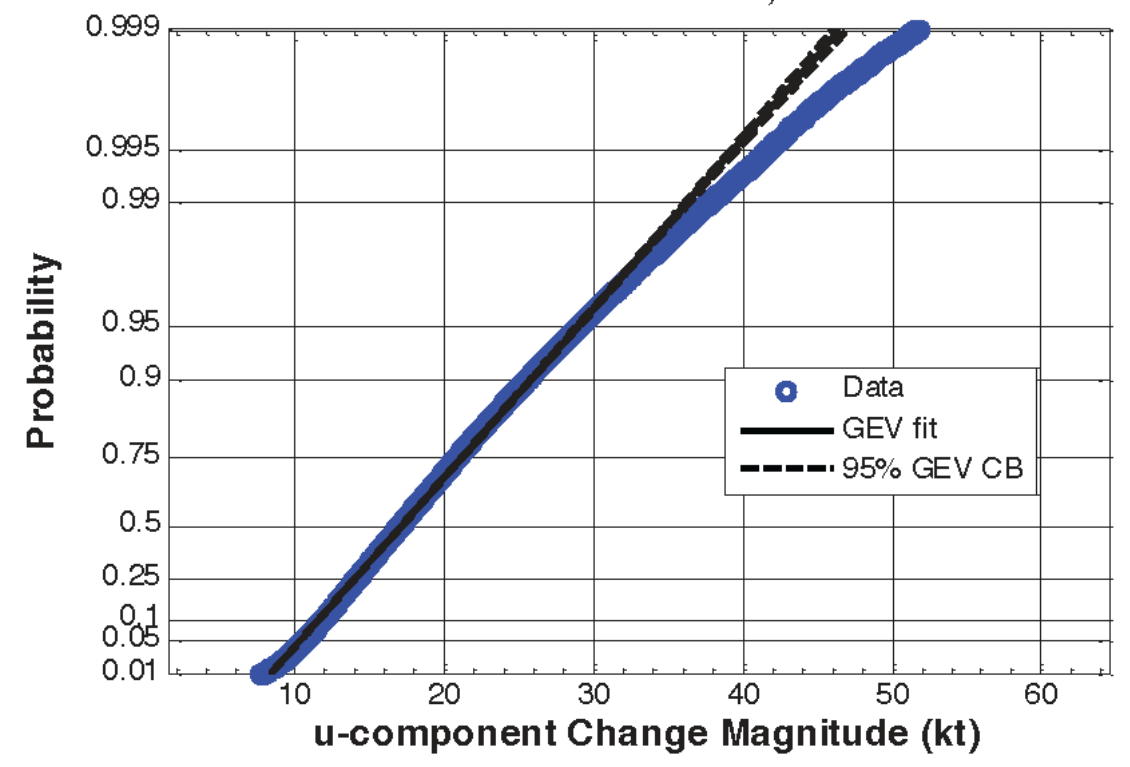

a)

ER Maximum 4-hr v-component Change Magnitudes with Generalized Extreme Value Fit and $95 \%$ Confidence Bounds, $n=276108$

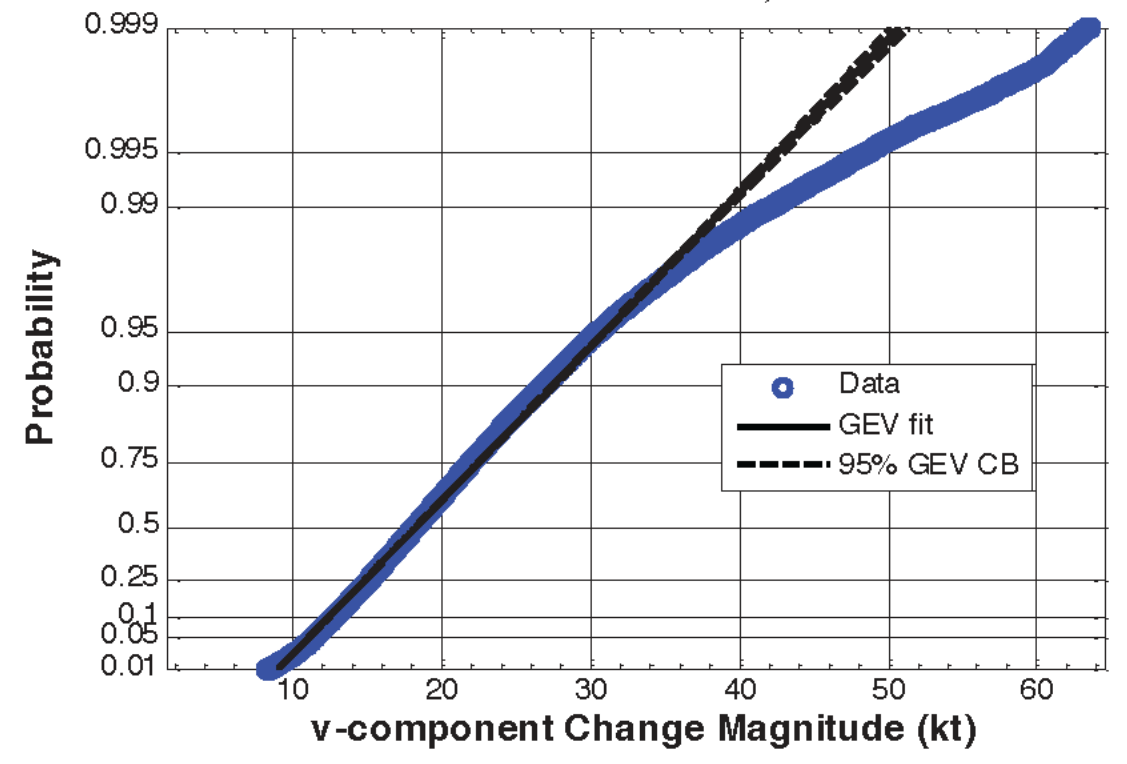

b)

Fig 4. ER maximum wind change from the 4-hour wind pairs with $95 \% \mathrm{CB}$ for the $\mathrm{u}$-(a) and vcomponent (b) wind changes. The magnitude of the wind component change is on the $x$-axis and probability is on the y-axis. The number (n) of pairs in the analysis is 276,108. 


\section{WR Maximum 4-hr u-component Change Magnitudes with Generalized Extreme Value Fit and 95\% Confidence Bounds, $\mathrm{n}=366$}

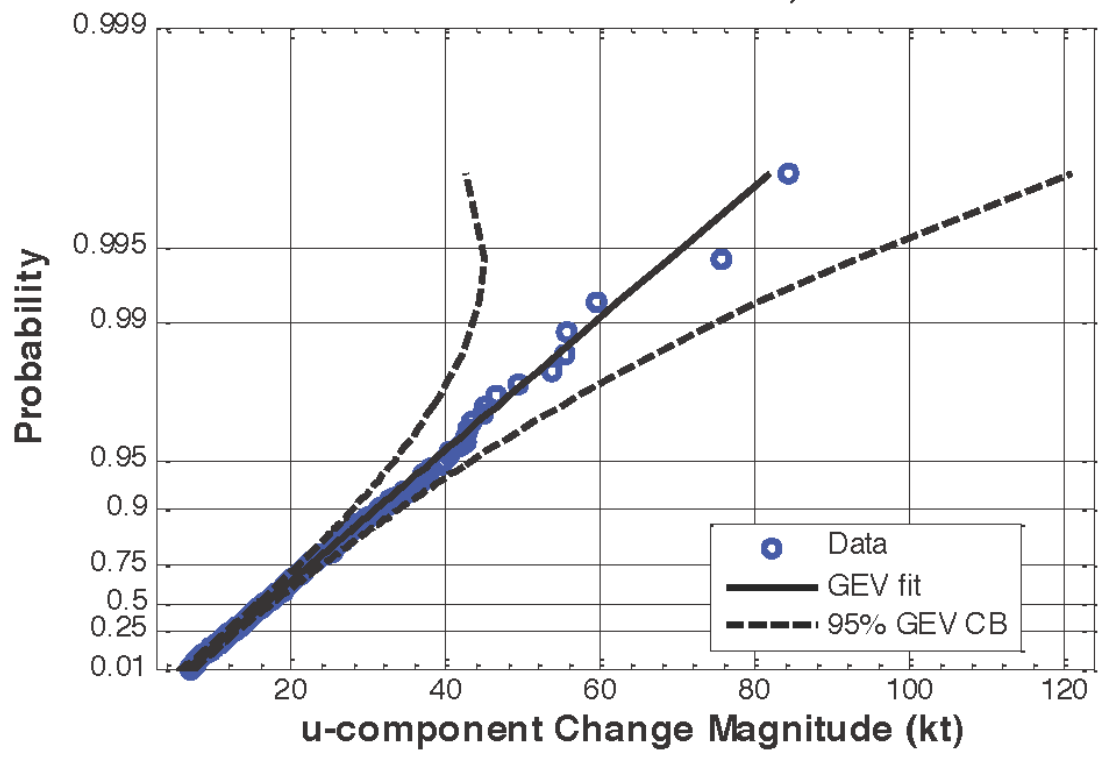

a)

WR Maximum 4-hr v-component Change Magnitudes with Generalized Extreme Value Fit and $95 \%$ Confidence Bounds, $\mathrm{n}=366$

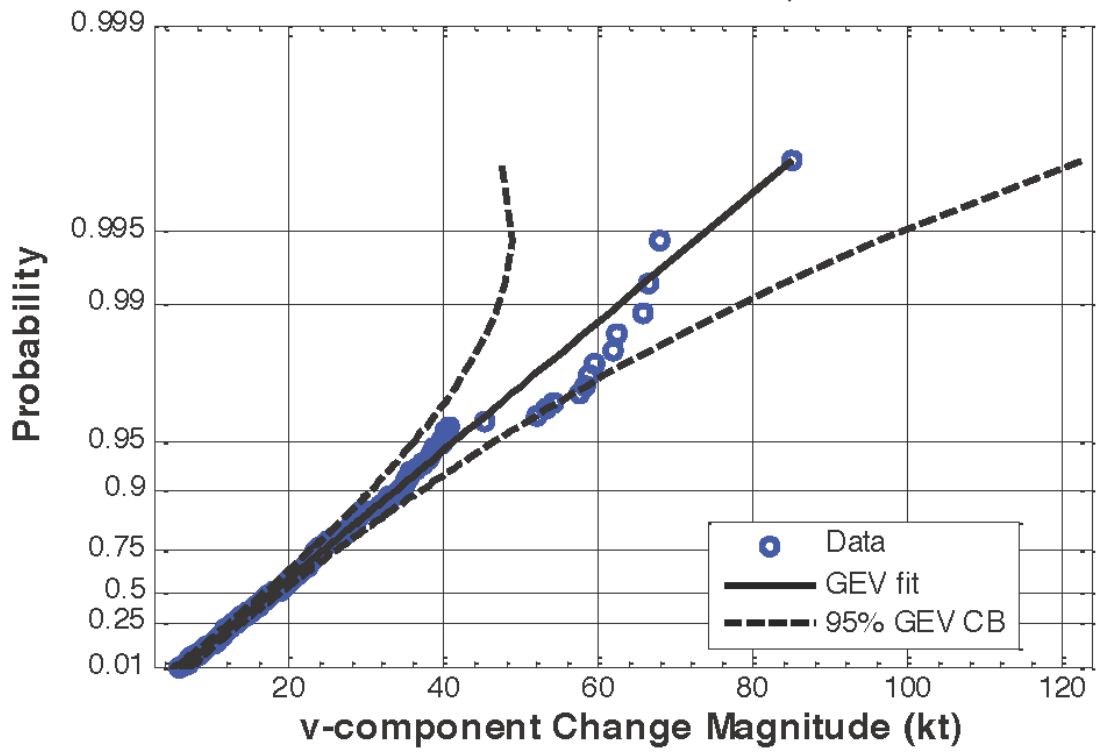

b)

Fig 5. WR maximum wind change from the 4-hour wind pairs with $95 \% \mathrm{CB}$ for the $\mathrm{u}-(\mathrm{a})$ and vcomponent (b) wind changes. The magnitude of the wind component change is on the $x$-axis and probability is on the $y$-axis. The number (n) of pairs in the analysis is 366. 


\section{WFF Maximum 4-hr u-component Change Magnitudes with Generalized Extreme Value Fit and $95 \%$ Confidence Bounds, $n=74$}

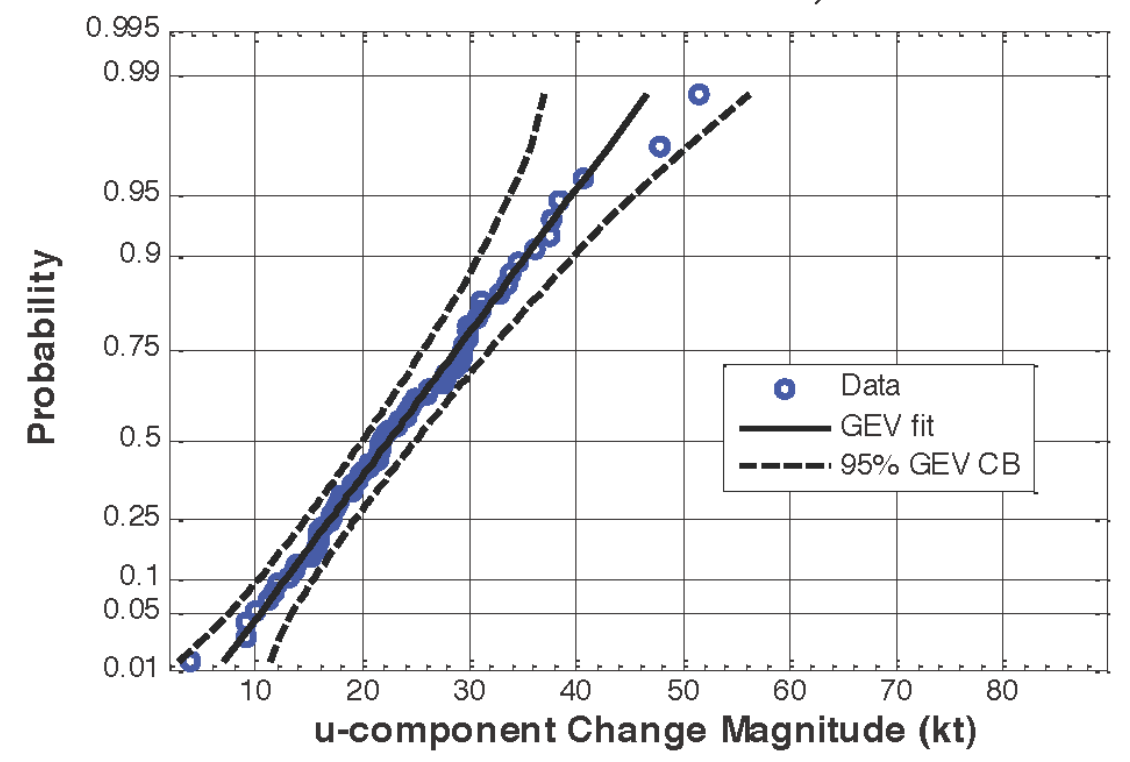

a)

WFF Maximum 4-hr v-component Change Magnitudes with Generalized Extreme Value Fit and $95 \%$ Confidence Bounds, $n=74$

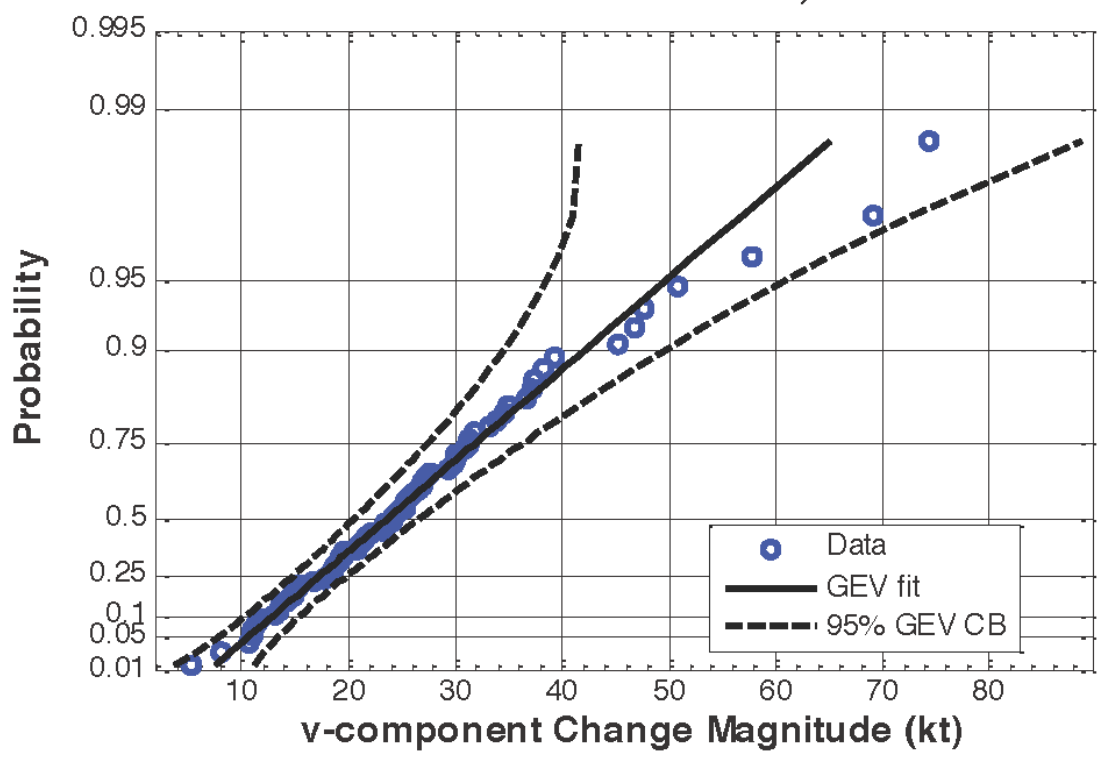

b)

Fig 6. WFF maximum wind change from the 4-hour wind pairs with $95 \% \mathrm{CB}$ for the u-(top) and vcomponent (b) wind changes. The magnitude of the wind component change is on the $x$-axis and probability is on the $y$-axis. The number (n) of pairs in the analysis is 74. 